\title{
Integrating Model-based Observer and Kullback-Leibler Metric for Estimating and Detecting Road Traffic Congestion
}

\author{
Abdelhafid Zeroual, Fouzi Harrou, Member, IEEE, Ying Sun, Nadhir Messai
}

\begin{abstract}
Efficient detection of traffic congestion plays an important role in the development of intelligent transportation systems by providing useful information for rapid decisionmaking. The aim of this study is to design an approach for road traffic congestion estimation and detection. Here, we design an innovative observer by integrating a hybrid piecewise switched linear traffic model (PWSL) with Luenberger observer estimator for enhanced road traffic density estimation. This observer termed PWSL-LO combines the flexibility of the PWSL model with the simplicity and efficiency of Luenberger observer to estimate the unmeasured traffic density. Moreover, this paper proposes an approach to monitor traffic congestion based on Kullback-Leibler distance (KLD) and exponential weighted moving average (EWMA) procedure. Residuals from the PWSLLO model are used as the input for KLD-EWMA scheme for congestion detection. This is motivated by the high capacity of KLD to quantitatively discriminate between two distributions. Here, the EWMA scheme is applied to the KLD measurements for congestion detection. Moreover, wavelet-based multiscale filter, a powerful feature/noise separation tool, is used to deal with the problem of measurement noise in the data. We evaluated the detection performance of this scheme by using traffic data from the four-lane SR-60 freeway in southern California. The proposed approach showed good abilities to estimate, monitor traffic congestions and to handle noisy traffic data.
\end{abstract}

Index Terms-Monitoring traffic congestion, Intelligent transportation systems, Kullback-Leibler metric, Luenberger observer.

\section{INTRODUCTION}

$\mathbf{T}$ RAFFIC flow congestion is rising gradually more and more in urban cities, which impacts negatively environment and economic developments. Thus, the development of intelligent transportation systems (ITSs) to mitigate congestion problems is becoming primordial [1]. Real-time traffic data are required to test, validate and improve ITS applications. For decades, various sensing technologies have been developed to collect traffic data. These technologies are mainly divided into two categories: Lagrangian sensors such as global position system GPS and probe vehicles, and conventional

A. Zeroual is with LAIG Laboratory, University of 08 May 1945, Guelma 24000, Algeria and CReSTiCURCA UFR SEN, University of Reims Champagne-Ardenne, Moulin de la Housse, BP 103951687 Reims Cedex 2, France

F. Harrou and Y. Sun are with King Abdullah University of Science and Technology (KAUST) Computer, Electrical and Mathematical Sciences and Engineering (CEMSE) Division, Thuwal, 23955-6900, Saudi Arabia e-mail: fouzi.harrou@kaust.edu.sa

N. Messai is with CReSTiC-URCA UFR SEN, University of Reims Champagne-Ardenne, Moulin de la Housse, BP 1039, 51687 Reims Cedex 2 France sensors such as magnetic loops, cameras, and radars [2], [3]. The implementation and the maintenance of these devices are costly in terms of labor and money. As an alternative, ITS approaches such as the traffic estimation allow reducing the physical components by using virtual sensors, which are inexpensive, accurate, reliable and more robust.

Traffic state estimation has a central role in several ITS applications and congestion monitoring. Traffic estimation consists mainly in reconstructing and estimating process of traffic variables, such as the density and traffic flow. It improves the quality of traffic data provided in real time based on limited quantities of measured sensors data.

In literature, there are two main approaches to traffic state estimation: model-based approaches and data-based approaches [4]. The detailed discussion of traffic state estimation techniques is given in [4]. Data-driven approaches are based on historical data to estimate the traffic state without the use of an explicit model. In [5], a statistical method based on the augmentation technique is proposed to estimate the traffic state. In [6], autoregressive integrated moving average using time series data is introduced for traffic estimation. Also, machine learning approaches are developed to address the complexity of traffic data and improve the robustness of observability [7], [8]. Other approaches used compressed sensors [9], deep learning [10], tensor-based method [11] and tensor completion algorithm [12] to improve both traffic state estimation and imputing performances.

On another hand, model-based state estimation approaches are based on analytical models describing the traffic dynamics. In literature, model-based estimation approaches have been shown good abilities in traffic estimation in terms of accuracy and the representation of several traffic phenomena [13], [14]. There are numerous model-based state estimation techniques in the literature, such as Kalman filtering (KF) and its extensions such as Duel extended KF [15] and mixture KF [16], particle filter [17] and adaptive smoothing filter [18]. However, these filtering estimation techniques require high computational time and cost [4]. Other model-based estimation approaches are also developed instead of filtering approaches. For instance, in [19] a mapping method has been proposed based on a phase transition model to estimate traffic variables using probe vehicle data. In [20], a distributed consensus approach has been developed for large-scale estimation.

In the literature, there is much discussion about traffic modeling. There are three main traffic modeling families: mi- 
croscopic, mesoscopic and macroscopic models. Microscopic modeling considers the individual description of vehicles, mesoscopic modeling considers the packets of vehicles sharing the same behaviors and finally the macroscopic modeling in which the dynamic of traffic flow is described as that of a fluid [2]. By comparing these modeling approaches, the macroscopic modeling presents fewer parameters, low computation times and provides a good description of traffic flow [21]. This paper presents a macroscopic model for traffic flow modeling.

Recently, hybrid modeling approaches have received much attention due to their ability to models traffic dynamics by considering several phenomena, such as congestion wave propagation [22], [23]. The piecewise switched linear (PWSL) traffic model is one of the most recent hybrid macroscopic models for traffic modeling [23]. It describes the traffic dynamics through the interaction of continuous traffic density evolution and the discrete event of congestion wave propagation. The PWSL is an extended and improved version of the switched mode model [22]. Specifically, it is a linearized and simplified version of the cell transmission model, which is commonly used in traffic modeling literature [21]. Of course, the PWSL model permits the description of several traffic phenomena which make it suitable for various applications of estimation, control, and monitoring [23]. This fact is mainly due to its flexibility in modeling, its simplicity of computation and facility to implementation.

Real-time knowledge of traffic state is a key element in designing ITSs. This paper integrates the PWSL model and Luenberger observer for accurately estimating road traffic density. In this approach, Luenberger observer is used as an estimator because of its simplicity of design, real-time implementation, ability to deal with missing measurements, and capacity to obtain suitable estimation performance [24], [25].

Timely detection of abnormal congestion provides useful information for rapid decision-making to reduce the number of accidents, reduce travel time, wasteful fuel consumption and pollution, and improve safety. Several detection approaches are developed for road congestion monitoring including sensor and vision-based approaches [26]. Sensor-based approaches usually use loop detectors that have the advantage of easy data collection and processing, and suitable for real-time application. However, Vision-based approaches use cameras and images, which require hard processing to extract data also a considerable computation time. Also, vision-based approaches are challenging to implement in real-time detection [26].

Furthermore, this paper proposes an efficient scheme based on Kullback-Leibler Divergence (KLD) to detect abnormal traffic congestion. Specifically, this approach uses the residuals from PWSL-LO model as the input to KLD-based detection scheme. Indeed, KLD is commonly used to compute the dissimilarity between two probability distributions, which make it very useful as an anomaly detection metric. The KLD distance between the distribution of residuals of the abnormal samples and the distribution of normal samples is much larger than the distance between the residuals of distribution of normal samples and the reference distribution of the training data (congestion-free data). In other words, KL distance becomes closer to zero under the congestion-free data, whereas a larger KL distance values are obtained in the presence of abnormal traffic congestion. Here, we introduce an approach that merges the KLD into the exponentially weighted moving average monitoring chart, termed KLD-EWMA. However, traffic data collected from sensors are usually noisy, which makes the congestion detection more challenging as the noisy data degrades detection performances. Multiscale filtering using wavelets is a powerful technique for features/noise separation [27], [28]. The objective is to exploit the advantages of both a multiscale filtering based on wavelet and of KLD-EWMA scheme to better detect congestions and to deal with the problem of measurement noise in the data.

In summary, this paper aims to contribute to the estimation and detection of traffic congestion. First, we develop a PWSLLO observer for modeling and estimating traffic density. Second, we develop KLD-based EWMA scheme for traffic congestion monitoring. Residuals from PWSL-LO observer are used as the input to fed KLD-based EWMA approach for detecting potential traffic congestion. The proposed KLDEWMA scheme aggregates all of the information from past and actual samples in the decision rule, which make it sensitive to abnormal traffic congestion. However, noisy measurements may degrade the quality of the detector performance by increasing the false alarms and missed detection. The measurement noise is distributed in the time-frequency domain which requires efficient feature extraction from time as well as frequency scale. Wavelet-based multi-scale representation of data has shown to be a powerful data analysis tool since it can extract useful data simultaneously from time as well as frequency domain. Multiscale filtering using wavelets is a powerful technique for features/noise separation. Third, we applied wavelet-based multiscale filtering on residuals from PWSL-LO for further enhanced detection results. We evaluate the efficiency of the proposed PWSL-LO-based KLD methodology on traffic data from the four-lane SR-60 freeway in southern California.

The following section presents related works on traffic monitoring approaches. Section III introduces a brief overview of traffic modeling and the PWSL model to design the proposed estimation method. Section IV review the proposed PWSLLO observer and its use in traffic estimation. We then present the proposed congestion detection approach in Section V. In Section VI, we assess the efficiency of the developed approach using via real traffic data, and Section VI concludes with a discussion.

\section{RELATED WORKS}

Over the last two decades, various estimation techniques have been developed. Model-based estimation methods showed good performance in parameters estimation. They are very helpful for estimating data that are difficult to measure [13]. However, these approaches depend on the accuracy of the model used [14]. Several filter-based traffic estimation 
approaches are proposed in the literature, including Kalman filters [29], such as extended Kalman, particle Kalman [30], and Monte Carlo mixture Kalman filters [31]. Recently, Zhang and Mao proposed using interactive multiple model filtering to estimate the traffic state and input variables [32]. This approach is based on Switched Mode Model (SMM), which takes into account only the fully free and fully congested modes. However, this approach is computationally time-consuming. Hidayat et al. designed an observer based on the transformation of a METANET traffic model to the Takagi-Sugeno fuzzy model [33]. Morarescu et al. proposed an estimation approach using the Luenberger observer and SMM, taking into account only the congestion wave direction. Other approaches rely on machine learning methods, such as neural network [34] and altricial-intelligent [35]. Such methods depend on the availability of quality input data, and their implementation is no easy task, especially for real-time applications.

To guarantee reliable traffic monitoring, researchers and engineers have developed several technologies. Most of the proposed approaches use information collected from cameras or sensors. Vision-based approaches are based on information extracted from images and videos for road traffic monitoring [26], [36], [37]. Mammeri and Zuo (2016) proposed a vision-based approach to detect pedestrians based on histograms of oriented gradient (HOG) and support vector machine algorithm [36]. Beauchemin et al. (2012) designed a vision-based driving assistance system for the analysis of driver intentionality [37]. In [38], Mehboob et al. introduce a vision-based traffic event detection technique by using fuzzy logic. In this approach, a fuzzy-based algorithm is used to discriminate between normal traffic and traffic congestion using several variables including traffic density, the speed of vehicles, and the number of vehicles per frame. Indeed, vision-based approaches are mainly based on images or object descriptors and require preprocessing times, elevate cost and complex installations [39]. Other approaches are mainly based on information captured by sensors, which provide real-time and do not require any pretreatment. In [40], Wang et al. introduced a sensor-based approach for freeway incident detection based on the partial least squares regression (PLSR). In this approach, a PLSR model is built based on the training data and then used to discriminate normal traffic from incidents event. Troiano et al. (2011) developed an approach to detect water and ice over road surfaces using sensor measurements [41]. Also, they developed an algorithm to estimate the state of the sensor at different conditions (dry, wet, or icy). Chan and Dillon (2013) proposed an on-road sensor configuration design for traffic flow prediction via fuzzy neural networks and Taguchi technique [42]. In this scheme, the Taguchi approach is used to select a suitable number of on-road sensors. Ali et al. (2012) presented a new method using multiple inductive loops to detect both small (e.g., bicycle) and large (e.g., bus) vehicles, which is a necessary requirement for sensing heterogeneous and lane-less traffic [43].

\section{Preliminaries}

The piecewise switched linear model (PWSL) is a macroscopic traffic model, which is driven from the macroscopic
Daganzo's Cell transmission model [23], [44]. It is a simple modified and linearised macroscopic version of CTM model. Generally speaking, the PWSL model describes the traffic dynamics through a set of linearised modes that summarized the several traffic statuses, according to the traffic congestion waves propagation movement directions.

Maximal flow $Q_{M, i}$, critical density $\rho_{c, i}$, free flow speed $v_{f, i}$, congestion wave speed $w_{i}$ and jam density $\rho_{j, i}$ are the essential parameters of the PWSL model and basic characterises of traffic dynamics system. They are approximated through the fundamental diagram (FD), which is a crucial component in traffic theory. The FD is an empirical curve representing the relationship between flow density, speed density, and flow speed. It has several forms, such as triangular, trapezoidal and parabolic. The triangular form is the most known and used in traffic modeling and applications because of its simplicity and its suitable representation of macroscopic parameters (Figure 1).

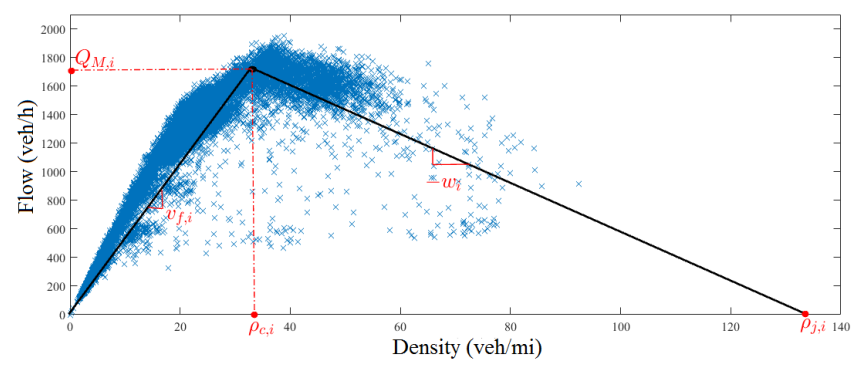

Fig. 1: FD triangular form

The PWSL model describes the traffic dynamics according to the switching between $2 \times N$ modes (for $N$ cells (Figure 2)). Each mode gives the dynamic of traffic density for the traffic statues of cells, respecting the congestion wave propagation movements (upstream propagation or downstream) that cross the road section. The switching between modes ensures around of the critical density of each cell. Figure 3 shows the evolution of PWSL and the propagation of congestion in cells for each mode. We admit that the cell presents the $F$ status if it is free $\left(\left(\rho_{i}(k)\right) \leq\left(\rho_{c, i}\right)\right)$ and $C$ status if it is congested $\left(\left(\rho_{i}(k)\right)>\right.$ $\left.\left(\rho_{c, i}\right)\right)$.

By considering the assumption of a non-uniform length of cells lengths, the traffic density dynamics in each cell can be expressed as follows:

$$
\rho_{i}(k+1)=\rho_{i}(k)+\frac{\Delta T}{\Delta x_{i}}\left(q_{i, \text { in }}(k)-q_{i, \text { out }}(k)\right),
$$

where $q_{i, \text { in }}(k)$ is the input flow to cell $i$ and $q_{i, o u t}(k)$ is the output flow of cell $i$ during $[k, k+1)$, including mainline flow, on-ramp flow and off-ramp flow (if they exist). $\Delta x_{i}$ and $\Delta T$ are respectively the length of cell $i$ and the step time. The flow in each cell $i$ is the minimum between the supply $\left(S_{i-1}(k)=\min \left(v_{i-1} \rho_{i-1}(k), Q_{M, i-1}\right)\right)$ and receive function $\left(R_{i}(k)=\min \left(Q_{M, i}, w_{i}\left(\rho_{j, i}-\rho_{i}(k)\right)\right)\right)$.

$$
q_{i}(k)=\min \left(S_{i-1}(k), R_{i}(k)\right),
$$




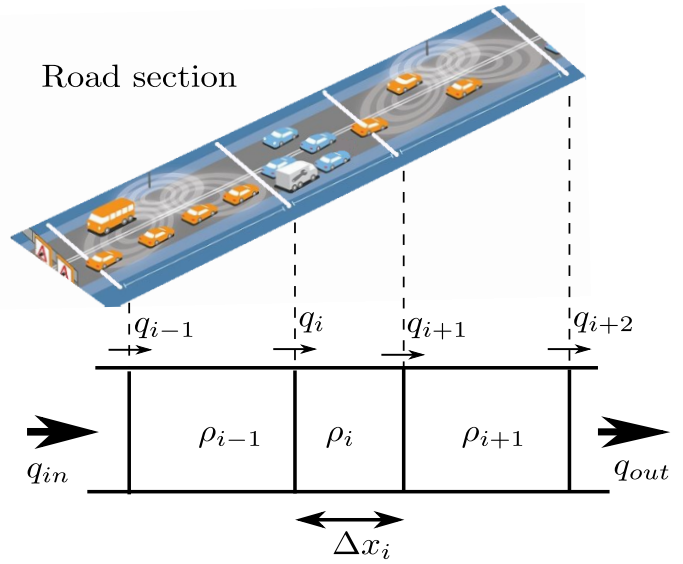

Spatial discretization

Fig. 2: Road section with spatial discretization.

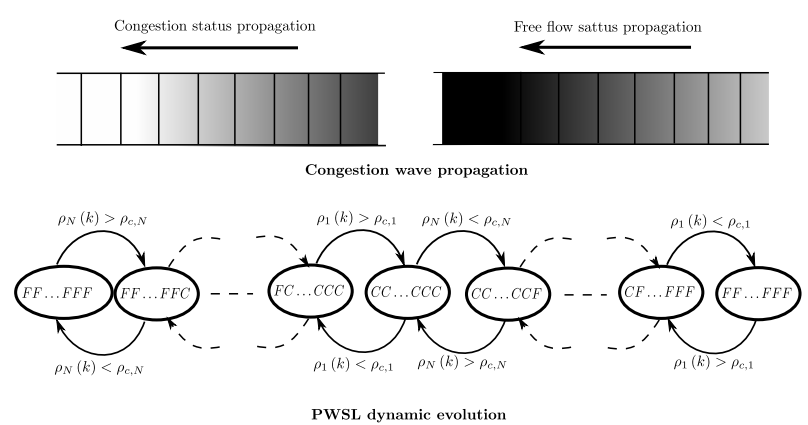

Fig. 3: PWSL traffic dynamic.

The supply and demand functions of boundary cells present deferent configuration than other cells and which are given by: cell 1:

$\begin{cases}S_{0}=q_{i n} & \text { if cell } i \text { is free, } \\ R_{1}=\min \left(Q_{M, 1}, w_{1}\left(\rho_{j, 1}-\rho_{1}(k)\right)\right) & \text { if cell } i \text { is not }\end{cases}$

cell $N$ :

$\begin{cases}S_{N-1}= & \min \left(v_{N-1} \rho_{N-1}(k), Q_{M, N-1}\right) \text { if cell } N \text { is free } \\ R_{N}= & \text { qut }\end{cases}$

The general form of the PWSL model in each mode is expressed as,

$$
\begin{gathered}
\rho(k+1)=A_{s} \rho(k)+B_{s} u(k)+D_{s} \\
D_{s}=B_{j a m, s} \rho_{j}+B_{Q, s} Q_{M} \\
y(k)=c \rho(k)
\end{gathered}
$$

where $s=\{1, \ldots, 2 N\}$ is the mode system index, $\rho(k)=\left[\rho_{1}(k), \ldots, \rho_{N}(k)\right]^{T}$ is the state vector, $u(k)=$ $\left[q_{\text {in }}(k), q_{O N, 2}(k), \ldots, q_{O N, N-1}(k), q_{\text {out }}(k)\right]^{T}$ is the entering flow vector (i.e., input, output, and on-ramp flow), $Q_{M}=\left[Q_{M, 1}, \ldots, Q_{M, N}\right]^{T}$ is the maximal flow vector, $\rho_{j}=$ $\left[\rho_{j, 1}, \ldots, \rho_{j, N}\right]$ is the jam density vector, $y(k)$ is the system output, and $\left(\rho(k), u(k), Q_{M}, \rho_{j}\right) \in \mathbb{R}^{N \times 1}, A_{s} \in \mathbb{R}^{N \times N}$, $B_{s} \in \mathbb{R}^{N \times N}, D_{s} \in \mathbb{R}^{N \times N}$, and $c \in \mathbb{R}^{2 \times N}$ are given matrices with structures that relies on the state of traffic in the considered road segment. More details about the PWSL model can be found in [23].

For the sake of statistical congestion detection, let us now focus on the case without the presence of congestion which is the case of free flow mode. The state representation of the PWSL in case of free-flow mode is given by (9) in which $s=1$ and $D_{1}=0$.

$$
\begin{gathered}
\rho(k+1)=A_{1} \rho(k)+B_{1} u(k) \\
y(k)=c \rho(k)
\end{gathered}
$$

For the configuration of the matrices $A_{1}$ and $B_{1}: \alpha_{i}=v_{f, i}$, $\delta_{i-1}=v_{f, i-1}, \gamma_{i, i \in[1, N-1]}=1$ and other parameters are equal to zero. The matrices $A_{1}$ and $B_{1}$ are given as

$$
A_{1=}\left[\begin{array}{ccccc}
1-\frac{\Delta T}{\Delta x_{1}} v_{f, 1} & 0 & 0 & \cdots & 0 \\
\frac{\Delta T}{\Delta x_{2}} v_{f, 1} & 1-\frac{\Delta T}{\Delta x_{2}} v_{f, 2} & 0 & \ddots & \vdots \\
0 & \ddots & \ddots & \ddots & 0 \\
\vdots & \ddots & & & 0 \\
0 & \cdots & 0 & \frac{\Delta T}{\Delta x_{N}} v_{f, N-1} & 1-\frac{\Delta T}{\Delta x_{N}} v_{f, N}
\end{array}\right]
$$

\section{OBSERVER DESIGN FOR PWSL FREE TRAFFIC MODEL ESTIMATION}

Generally, observers use the measured system variables to estimate the unmeasured system state vector and reconstruct its outputs from the estimated state. Here, we merge the advantages of the PWSL model and model-based estimation using Luenberger observer to enhance the estimation of traffic density. The key concept of Leuenberger observer is the estimation of the state vector by the adjustment and the correction of the system from the estimated error [24], [25]. It is widely used in control and estimation because of its flexibility, computational efficiency, and facility to implement. The herein proposed approach is based on estimating the state vector using past measurements along a given road portion segment. This approach provides a suitable real-time estimation and prediction of the traffic density characteristics.

Figure 4 shows the general structure of the proposed observer for traffic data estimation. First, the observer is initialized with initial values and then used to estimate density dynamics in a given road section using the provided boundary information, such as the input and output flow of the considered road section.

The equation of the observer is given as follows:

$$
\begin{gathered}
\hat{\rho}(k+1)=A_{1} \hat{\rho}(k)+B_{1} u(k)+L(y(k)-\hat{y}(k)) \\
\hat{y}(k)=c \hat{\rho}(k)
\end{gathered}
$$




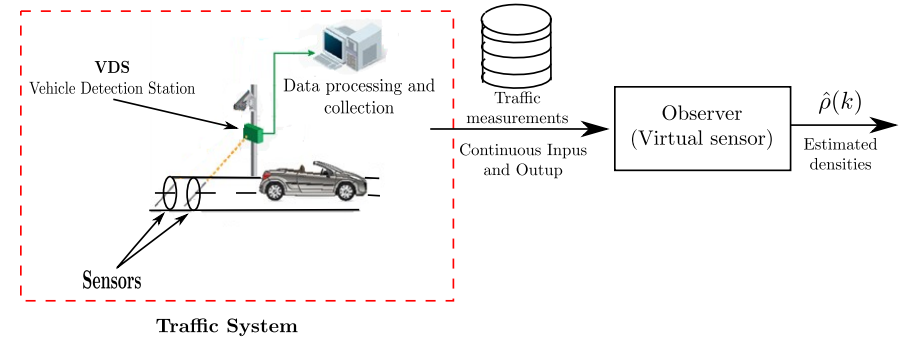

Fig. 4: Traffic density estimation structure.

where $L(y(k)-\hat{y}(k))$ is the correction term, in which $L$ is the observer gain that leads to minimise the estimation error (13) and tend it to zero.

$$
e(k)=\rho(k)-\hat{\rho}(k)
$$

The observation error dynamics can be expressed as follows:

$$
e(k+1)=\rho(k+1)-\hat{\rho}(k+1)=\left[A_{1}-L c\right] e(k)
$$

The condition of convergence of the estimation error can be guarantee by the existence of Lyapunov function $V(k, e(k))$, where

$$
V(k, e(k))=e(k)^{T} P e(k)>0 .
$$

For which the variation $\Delta V(k, e(k))$ is negative definite

$$
\Delta V(k, e(k))=V(k+1, e(k+1))-V(k, e(k))<0,
$$

where $P$ is a symmetric positive definite matrix.

To ensure the asymptotic stability of the density estimation error dynamics, Lyapunov function can be established by solving a linear matrix inequality (LMI) through the following theorem.

Theorem 1: The estimated density converges asymptotically with the reel density if there exist:

1) A Lyapunov function $V(k, e(k))$ of negative variation and

2) A symmetric matrix $P$ satisfy the following LMI,

$$
\left[\begin{array}{cc}
-P & A_{1}^{T} P-c^{T} U^{T} \\
P A_{1}-U c & -P
\end{array}\right]<0
$$

where $U=P L$.

Proof 1: We consider that there exists a Lyapunov function $V(k, e(k))$ with a negative variation $\Delta V(k, e(k))$ for which

$$
\begin{aligned}
\Delta V(k, e(k)) & =V(k+1, e(k+1))-V(k, e(k)) \\
& =e(k+1)^{T} \operatorname{Pe}(k+1)-e(k)^{T} P e(k) \\
& =e(k)^{T}\left(\left[A_{1}-L c\right]^{T} P\left[A_{1}-L c\right]-P\right) e(k) .
\end{aligned}
$$

Hence,

$$
\left[A_{1}-L c\right]^{T} P\left[A_{1}-L c\right]-P<0
$$

After the application of the Schur complement formula to (18), we obtain:

$$
\left[\begin{array}{cc}
-P & {\left[A_{1}-L c\right]^{T} P} \\
P\left[A_{1}-L c\right] & -P
\end{array}\right]<0 .
$$

By putting $U=P L$ the inequality (19) is rewritten as:

$$
\left[\begin{array}{cc}
-P & A_{1}^{T} P-c^{T} U^{T} \\
P A_{1}-U c & -P
\end{array}\right]<0
$$

\section{A LINEAR OBSERVER-BASED KLD FOR CONGESTION DETECTION}

\section{A. KLD-based congestion detection scheme}

In this section, we present an efficient anomaly detection scheme using the Kullback-Leibler distance (KLD). The KLD is a well-known metric to quantitatively measure the distance between two probability distributions. This metric has been extensively used in several fields including information theory, statistical inference, and data mining.

The Kullback-Leibler information between two probability density functions (PDFs) $p_{1}(x)$ and $p_{2}(x)$ is defined as

$$
I\left(p_{1}: p_{2}\right)=\int_{\mathbb{R}^{d x}} p_{1}(x) \log \left[\frac{p_{1}(x)}{p_{2}(x)}\right] d x,
$$

and between $p_{2}(x)$ versus $p_{1}(x)$ is given by:

$$
I\left(p_{2}: p_{1}\right)=\int_{\mathbb{R}^{d_{x}}} p_{2}(x) \log \left[\frac{p_{2}(x)}{p_{1}(x)}\right] d x,
$$

which is nonsymmetric (i.e., $\left.I\left(p_{1}: p_{2}\right) \neq I\left(p_{2}: p_{1}\right)\right)$ and nonnegative (i.e., $I\left(p_{1}: p_{2}\right) \geq 0$ and $I\left(p_{2}: p_{1}\right) \geq 0$ ).

The KLI measure is asymmetric, which make the threshold selection for congestion detection challenging. To bypass this difficulty, the KL distance (KLD), which is a summarized version of KLI is used here [45]. The KLD between $p_{1}(x)$ and $p_{2}(x)$ is defined as:

$$
\begin{aligned}
K L D\left(p_{1} ; p_{2}\right) & =I\left(p_{1}: p_{2}\right)+I\left(p_{2}: p_{1}\right), \\
& =\int_{\mathbb{R}^{d_{x}}}\left(p_{1}(x)-p_{2}(x)\right) \log \left[\frac{p_{1}(x)}{p_{2}(x)}\right] d x,
\end{aligned}
$$

Let us consider the particular case when $p_{1}(x)$ and $p_{2}(x)$ are Gaussian, $p_{1} \sim \mathcal{N}\left(\mu_{0}, \sigma_{0}\right)$ and $p_{2} \sim \mathcal{N}\left(\mu_{1}, \sigma_{1}\right)$, where $\mu_{0}$ and $\mu_{1}$ are the means and $\sigma_{0}^{2}, \sigma_{1}^{2}$ are the variances for $p_{1}$ and $p_{2}$, the closed form of KLD is [46],

$$
\begin{aligned}
K L D\left(p_{1} \backslash \backslash p_{2}\right)= & \frac{1}{\sigma_{0} \sqrt{2 \pi}} \int \exp \left(\frac{\left(x-\mu_{0}\right)^{2}}{2 \sigma_{0}^{2}}\right) \\
& {\left[\log \frac{\sigma_{1}}{\sigma_{0}}-\frac{\left(x-\mu_{0}\right)^{2}}{2 \sigma_{0}^{2}}+\frac{\left(x-\mu_{1}\right)^{2}}{2 \sigma_{1}^{2}}\right] d x } \\
= & \frac{\left(\mu_{1}-\mu_{0}\right)^{2}}{2 \sigma_{1}^{2}}+\frac{1}{2}\left(\log \frac{\sigma_{1}^{2}}{\sigma_{0}^{2}}+\frac{\sigma_{0}^{2}}{\sigma_{1}^{2}}-1\right) .(25)
\end{aligned}
$$

When $p_{1}(x)$ and $p_{2}(x)$ are similar, KLD is around zero due measurement noise and uncertainty. Otherwise, large values of KLD reflect a great deviation between the two distributions. This makes KLD very useful in anomaly detection framework. Here, we used KLD as an anomaly indicator. In absence of anomalies, KLD becomes closer to zero, whereas a larger KLD value is obtained under the presence of anomalies that lead to the presence of a potential congestion. 


\section{B. Combination of KLD and EWMA scheme for congestion detection:}

This section briefly describes the proposed PWSL-LO-based KLD-EWMA congestion detection scheme. The proposed approach merges PWSL-LO observer and the KLD metric with EWMA scheme to separate normal from abnormal features. We first construct an appropriate observer-based estimator under free-flow traffic conditions (i.e., mode 1). Then, this model is applied to inspect traffic for abnormal flow by looking for future deviations from normality. PWSL-LO residual sequences have been used as an anomaly detection index. Anomaly decision statistic has been designed using KLD as a measure between PDFs of congestion-free residuals and actual residual then mixed with EWMA chart for congestion detection. Under the congestion-free scenario, KLD is close to zero due to measurement noise and uncertainty. However, a significant departure from zero of KLD indicates important changes from normal conditions. The main advantage of combining KLD and EWMA for congestion detection is that the EWMA scheme aggregates all available information from earlier and actual samples in the decision rule [47]. Defining the vector of KLD measurements as computed in (22): $K L D=\left[d_{1}, \ldots, d_{j}, \ldots, d_{n}\right]$. The KLD-EWMA congestion decision statistic is computed as follows:

$$
z_{t}^{K L D}=\nu d_{t}+(1-\nu) z_{t-1}^{K L D},
$$

where the initial value, $z_{0}^{K L D}$ is the anomaly-free mean of KLD vector, $\mu_{0}^{K L D} . \nu(0<\nu \leq 1)$ is a smoothing parameter. Generally, the selection of small values of $\nu$ is suitable for detecting small shifts in the mean of residuals (i.e., more weight is given to past observations), while the selection of higher values of $\nu$ is suitable to detect larger changes in the residuals mean. A value of $\nu$ is commonly selected between 0.2 and 0.3 to detect small and moderate changes [48]. When the KLDEWMA statistic, $z_{t}^{K L D}$, is beyond the decision threshold, $h$, then we claim that there is a congestion. Otherwise, the supervised process is considered in control. The KLD-EWMA congestion detection threshold is designed as [47],

$$
h=\mu_{0}^{K L D}+\kappa \sigma_{0}^{K L D} \sqrt{\left(\frac{\nu}{(2-\nu)}\left[1-(1-\nu)^{2 t}\right]\right.},
$$

where $\kappa$ is a multiplier of standard deviation of KLD, $\sigma^{K L D}=\sigma_{0}^{K L D} \sqrt{\left(\frac{\nu}{(2-\nu)}\left[1-(1-\nu)^{2 t}\right]\right.}$. When a univariate EWMA chart is utilized to detect abnormal measurements in a single variable, then the univariate EWMA chart usually has two parameters involved (i.e., $\nu$ and $\kappa$ ).

Traffic data gathered from sensors are usually noisy which makes congestion detection more difficult. The wavelet-based multiscale filter can provide effective noise/feature separation [27]. The novelty of our approach is to develop a robust congestion detection approach to noise measurements by exploiting the benefits of the multiscale representation of data and those of the KLD-EWMA scheme to better detect abnormal congestions.

As illustrated in Figure 5, to detect abnormal congestion: (1) we exploit the flexibility of the proposed PWSL-LO observer to extract important features from the traffic data and generate residuals, (2) we filter the generated residuals via wavelet multiscale filtering, and (3) we apply the KLD-EWMA scheme on the denoised residuals to detect potential traffic congestion.

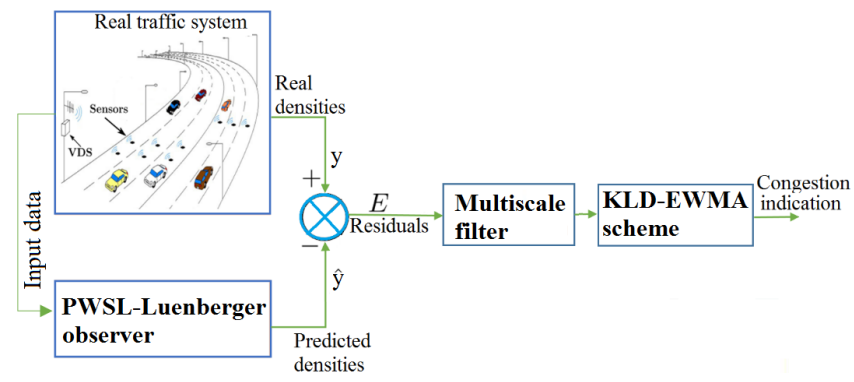

Fig. 5: A diagrammatic representation of the proposed procedure.

\section{CASE STUdy}

To illustrate the estimation and detection performance of the proposed methodology, we used real data from a highway portion of 1.73 miles of SR60 Highway, California. This road portion is beginning from San Antonio Avenue and ends to San Grove Avenue. It provides interesting congestion periods and it is equipped with the necessary magnetic loops for collecting traffic data. The chosen road portion is uniformly split into four cells of 0.43 miles (Figure 6). Each cell contains a loop detector.
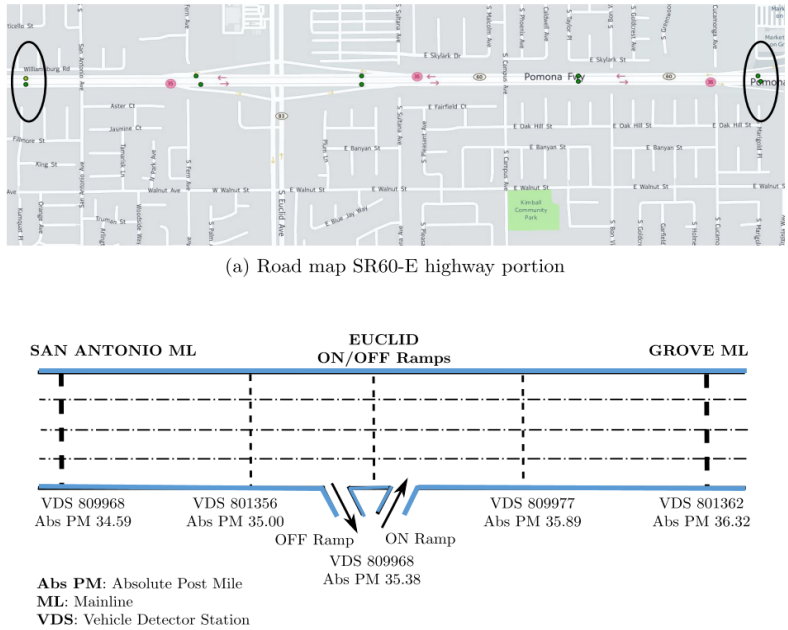

(b) Schematic representation of the SR60-E portion

Fig. 6: Road map and schematic representation of State Route 60 (SR 60) freeway.

To identify and approximate the PWSL model parameters, the calibration of FD, which is a crucial task in macroscopic models validation, is required [49]. A sampling time of five minutes for a row VDS data is used for 98 days (i.e., 28225 data points) to guarantee the availability of enough measurements of congested traffic. For further accuracy, we consider that each cell has its own FD and assume $99 \%$ functionality of the detectors.

We applied the calibration methodology given in [23], [50] to the considered road segment for identifying the triangular 
FD form and estimating the parameters of the PWSL model for each cell (Table I).

TABLE I: Values of the estimated FD parameters of the SR60 road section 6 .

\begin{tabular}{l|l|l|l|l|l}
\hline VDS & $\Delta x(m i)$ & $v_{f}(\mathrm{mi} / \mathrm{h})$ & $w(\mathrm{mi} / \mathrm{h})$ & $\rho_{c}(\mathrm{veh} / \mathrm{mi})$ & $Q_{M}(\mathrm{veh} / \mathrm{h})$ \\
\hline 809968 & 0.41 & 64.1481 & 30.7717 & 25.8819 & 1557 \\
\hline 801356 & 0.38 & 58.9681 & 26.4288 & 31.7438 & 1686 \\
\hline 801358 & 0.51 & 62.4373 & 18.6045 & 30.7691 & 1664,5 \\
\hline 809977 & 0.43 & 53.8030 & 18.6371 & 34.0653 & 1833 \\
\hline
\end{tabular}

To design the observer, we first consider that the initial value of the observer is $\left[\begin{array}{llll}0 & 0 & 0 & 0\end{array}\right]^{T}$, and that the observer is initially in free-flow status mode (mode 1). The observation matrix is given by

$$
c=\left[\begin{array}{llll}
1 & 0 & 0 & 0 \\
0 & 0 & 0 & 1
\end{array}\right] .
$$

The observer gains obtained by solving LMI (17) are

$$
L=\left[\begin{array}{cccc}
0.1308 & 0.9378 & 0 & 0 \\
0 & 0 & 0 & 0.3049
\end{array}\right]^{T}
$$

Figure 7 (a-d) shows the continuous estimation results for each cell of the road segment in free-flow statues during two days with the step time of $20 \mathrm{sec}$. Also, the scatter graph of the measured versus the estimated traffic density from PWSL-LO model are displayed in Figure 8. The results indicate that the PWSL-LO approach succeeds to appropriately estimate traffic density measurements.
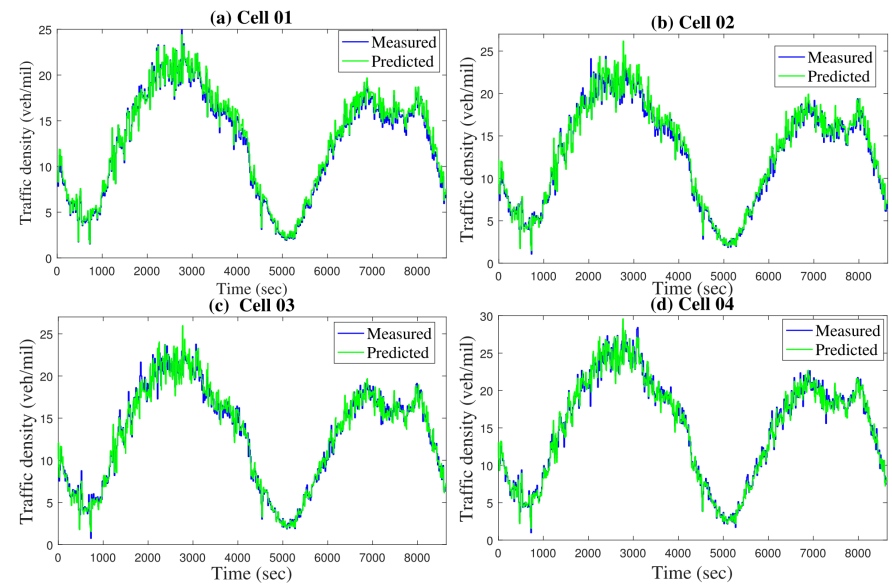

Fig. 7: The measured and the estimated traffic density using the PWSL-LO estimation approach of four different cells from the SR-60 dataset.

Next, the accuracy performances of the proposed PWSLLO approach are compared to that obtained using standalone PWSL and the cell transmission model (CTM), which is one of the most popular discrete macroscopic models in the literature (Table II). Results in Table II indicate that the PWSL-LO approach outperforms the standalone PWSL and CTM approaches. The result shows that the PWSL-LO model provides the smallest MAPE and RMSE values and highest $r^{2}$ values compared to that obtained with PWSL and CTM models (see Table II).
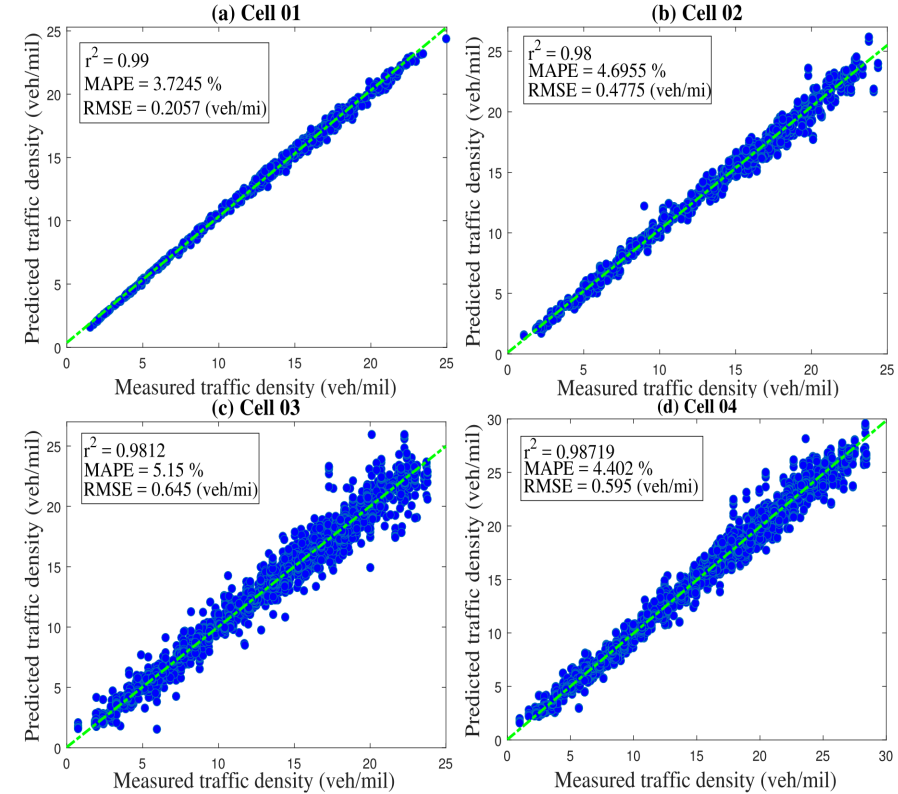

Fig. 8: Scatter graph of the measured versus the estimated traffic density obtained from the PWSL-LO estimation approach of four different cells from the SR-60 dataset.

\section{A. Detection results}

1) Detection of abrupt congestions: In this study, congestion detection performance has been characterized using missed detection rate (MDR) and false alarm rate (FAR) [51]. For a given congestion detection approach, the ideal situation is that its MDR value and its FAR value are small. Here, we evaluate the effectiveness of the KLD-EWMA algorithm in detecting abrupt congestion. We compared the proposed KLDEWMA algorithm to the conventional EWMA algorithm. We introduced a bias to the testing data from sample times 2500 to 3000 . The amplitude of the introduced bias is equal to $10 \%$ of the total variation found in the raw data. This scenario can result due to traffic jam during working hours. EWMA chart with $\nu=0.25$ and $386 \kappa=3$ is depicted in Figure 9(a). From Figure 9(a), we see that EWMA chart detects this congestion, but it resulted in many false alarms (i.e., FAR $=10.26 \%$ ). The results from the KLD-EWMA scheme with $(\nu=0.25$ and $\kappa=3$ ) are shown in Figure 9(b). Decision threshold has been computed using (27). In this example, the proposed scheme detected this abnormal congestion with $\mathrm{FAR}=1.17 \%$ and $\mathrm{MDR}=4 \%$. The KLD-EWMA scheme, when applied to denoised residuals using a wavelet-based filter, is illustrated in Figure 9(c). It can be seen that the KLD-EWMA algorithm using the filtered residuals can correctly this congestion.

Traffic data gathered from sensors are usually contaminated with noise, which makes the detection of abnormal congestion more difficult as noisy data degrades congestion detection quality. The level of noise relies on many factors such as weather conditions and road state. To show the robustness of the proposed approach to noise measurements, in this scenario we used noisy data and introduced the same abrupt congestion as in the first example (see Figure 10). The results of EWMA scheme based on the testing data is shown in Figure 10(a). 
TABLE II: Performance comparison of PWSL-LO, PWSL, and CTM approaches.

\begin{tabular}{l|l|l|l|l||l|l|l|l|l||c|c|c|c}
\hline & \multicolumn{3}{|c|}{ PWSL-based obsever with SR60 data } & \multicolumn{3}{c||}{ CTM model with SR60 data } & \multicolumn{3}{c}{ PWSL model with SR60 data } \\
\hline & Cell 01 & Cell 02 & Cell 03 & Cell 04 & Cell 01 & Cell 02 & Cell 03 & Cell 04 & Cell 01 & Cell 02 & Cell 03 & Cell 04 \\
\hline$r^{2}$ & 0.99 & 0.98 & 0.9812 & 0.9871 & 0.8321 & 0.8554 & 0.9531 & 0.9661 & 0.99 & 0.9623 & 0.9676 & 0.9672 \\
\hline RMSE (veh/mi) & 0.2057 & 0.4775 & 0.645 & 0.595 & 2.3426 & 2.2268 & 1.2682 & 1.2553 & 0.5242 & 1.1369 & 1.0545 & 1.2348 \\
\hline MAPE \% & 3.7245 & 4.6955 & 5.15 & 4.402 & 18.3618 & 16.6934 & 8.6984 & 7.2500 & 4.3084 & 8.3094 & 7.1736 & 7.4002 \\
\hline
\end{tabular}

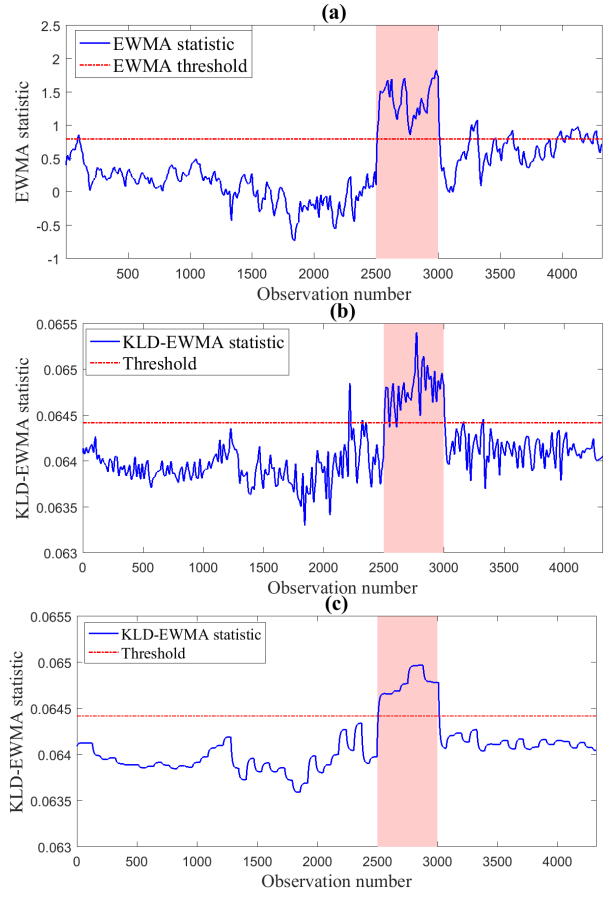

Fig. 9: Monitoring results from EWMA chart (a), KLDEWMA approach using the raw residuals (b), and the denoised residuals (c) in the case of abrupt congestion ( $10 \%$ bias), nonnoisy data.

Results indicate the ability of the EWMA scheme in detecting this abnormal trafc congestion but with several false alarms and missed detections (i.e., $\mathrm{MDR}=8.78 \%$ and $\mathrm{FAR}=19.87 \%$ ). Figure 10(b) shows detection results of the KLD-EWMA algorithm when using the raw residuals. From Figure 10(b), we see that the KLD-EWMA algorithm when using the raw residuals detect this congestion but with some false alarms and missed detections (i.e., FAR $=4.71 \%$ and $\mathrm{MDR}=14.40 \%$ ). In this study, to reduce the negative effect of noise on congestion detection quality we applied the KLD-EWMA approach to denoised residuals by wavelet (Figure 10(c)). Then, the KLDEWMA approach is shown in Figure 10(c), from which it can be seen that the FAR and MDR have been notably reduced. Of course, the wavelet-based denoising procedure applied to residuals enhanced significantly the detection quality of the KLD-EWMA approach by reducing the FAR and MDR.

Now, let us consider the scenario when the magnitude of the introduced bias is 5\% occurs between samples 2500 and 3000 into the testing noisy data. In this scenario, the monitoring results of the EWMA and KLD-EWMA approaches are depicted in Figure 11. The EWMA approach is shown in Figure 11(a). The EWMA is inefcient in detecting a relatively small level of congestion (i.e., MDR $=25.55 \%$ and FAR $=19.79 \%$ ). From

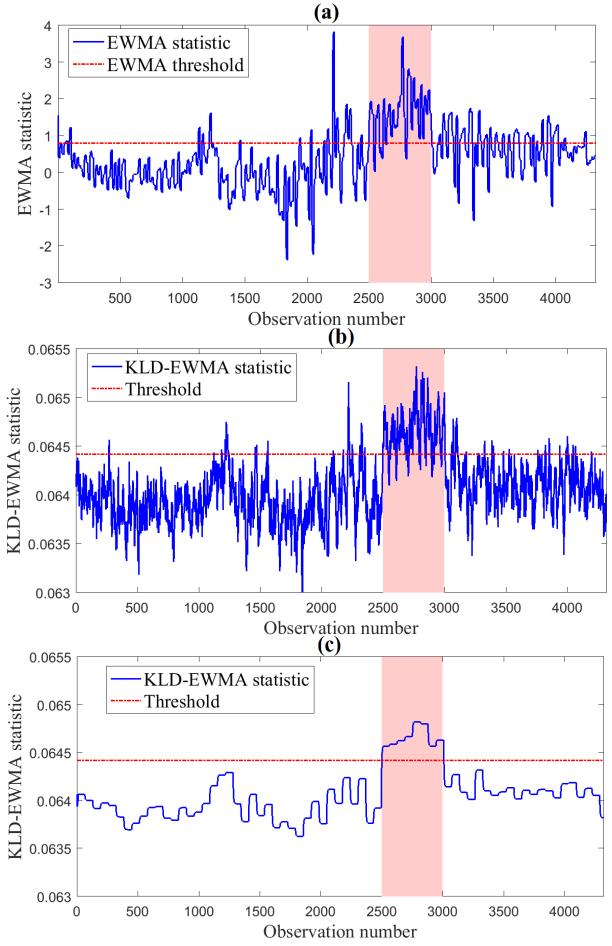

Fig. 10: Monitoring results from EWMA chart (a), KLDEWMA approach using unfiltered residuals (b), and the denoised residuals (c) in the case of abrupt congestion $(10 \%$ bias), non-noisy data example.

Figure 11(b), the KLD-EWMA approach detects this abnormal congestion with $\mathrm{FAR}=7.59 \%$ and $\mathrm{MDR}=26 \%$. On the other hand, The KLD-EWMA approach applied to filtered residuals detects correctly the introduced bias (Figure 11(c)). From this scenario, it can be seen that the detection capability of the KLD-EWMA scheme would decrease if the data is noisy and the congestion is relatively small. Also, It can be pointed out that the KLD-EWMA approach applied to filtered residuals by a wavelet-based multiscale filter is quite robust to the noise measurements.

2) Scenarios with intermittent congestion: In this scenario, to show the effectiveness of the proposed methodology in detecting intermittent congestion, intermittent congestion has been introduced into the raw data. Specically, the testing measurements have been contaminated with a bias of amplitude $5 \%$ of the total variation of raw measurements for time instant 1000 to 1200 , and bias of amplitude $10 \%$ for time instant 2500 to 3000. Monitoring results based on filtered an unfiltered data are shown in Figure 12(a-c). The EWMA approach shows very poor congestion detection performance (i.e., $\mathrm{MDR}=32.05 \%$ and FAR $=20.31 \%$ ). We note a significant improvement can be reached when applying the KLD-EWMA scheme to the 

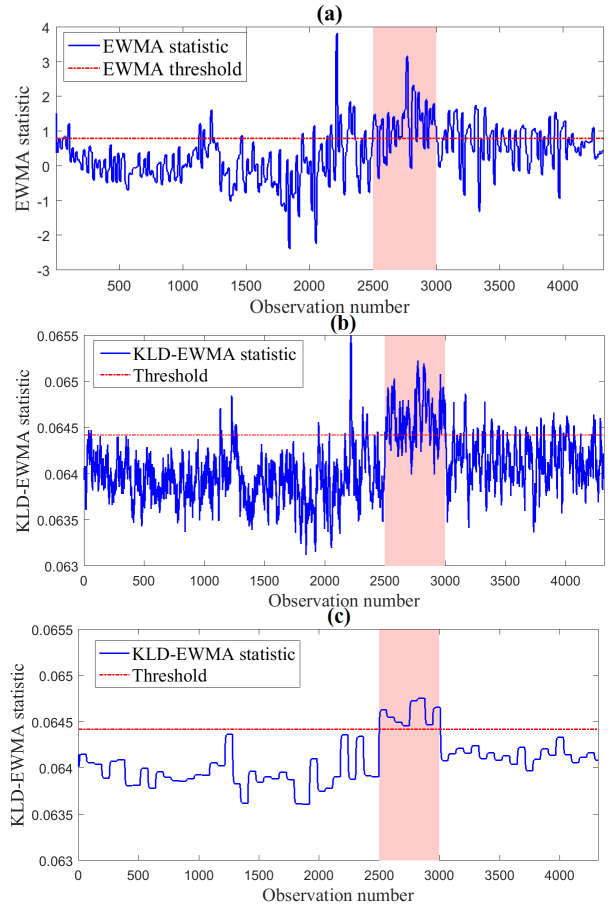

Fig. 11: Monitoring results from EWMA chart (a), KLDEWMA approach using the raw residuals (b), and the denoised residuals (c) in the case of abrupt congestion ( $5 \%$ bias), nonnoisy data example.

denoised residuals using wavelet-based multiscale filtering. It results in reducing the FAR form $5.05 \%$ to $0.53 \%$.
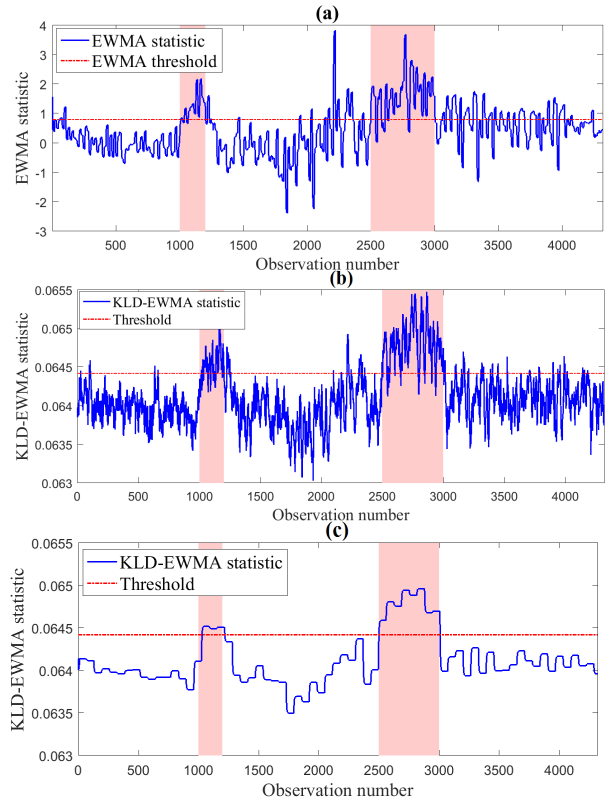

Fig. 12: Monitoring results from EWMA chart (a), KLDEWMA approach using the raw residuals (b), and the denoised residuals by wavelet (c) in the case with intermittent congestions, non-noisy data example.

3) Detection of gradual congestion: Here, gradual congestion, which is usually characterized by a slow increase of traffic flow, is considered to show the effectiveness of the proposed methodology. Gradual congestion scenarios may result from a slow increase in traffic flow due to bad weather conditions or increasing traffic demands. In this scenario, we observe a drift with a slope of 0.01 , compared to raw traffic measurements from the 3000 of the time instant. Figure 13(a) indicates that the EWMA approach detected a congestion at sample 3090 , but with few false alarms, FAR $=1 \%$. Figure $13(\mathrm{~b}-\mathrm{c})$ shows results of the KLD-EWMA scheme when applied to raw and denoised residuals, respectively. From Figure 13(b), the KLD-EWMA approach generates the first signal of congestion at the 3092 time instant, but with some false alarms (i.e., FAR $=1.6 \%$ ). The proposed approach, when applied to filtered data, gives a signal of congestion at the 3087 time instant without false alarms (Figure 13(c)).
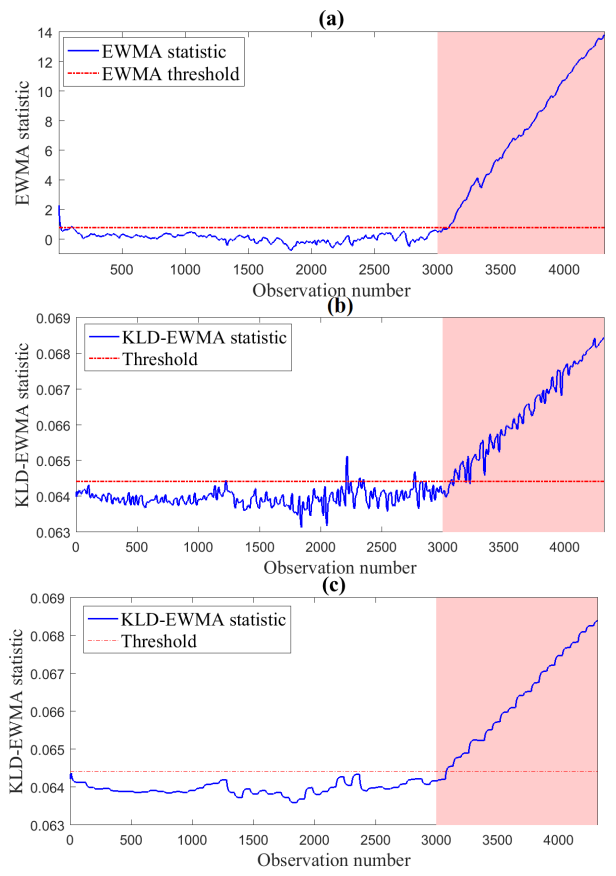

Fig. 13: Monitoring results from EWMA scheme (a), KLDEWMA scheme using the raw residuals (b) and the denoised residuals (c) in the case of gradual traffic congestion, nonnoisy data example.

Next, we assess the capacity of the proposed approach to monitoring gradual congestion in the case of noisy data. Figure 13(a) illustrates that the EWMA scheme detects the congestion at the 3052 observation with high false alarm rate (i.e., FAR=12.87\%). The KLD-EWMA chart is shown in Figure 14(b) when applied to unfiltered residuals, from which it can be seen that the first signal is given at the 3058time point but it does not stay stable above the threshold and results in many false alarms $(\mathrm{FAR}=3.4 \%)$. The KLDEWMA approach using the filtered residuals is shown in Figure 14(c). Figure 14(c) indicates that the KLD-EWMA scheme detects congestion approximately at the 3080 time instant without false alarms. Therefore, besides its sensitivity to small changes, the KLD-EWMA chart with wavelet multiscale filter is also robust to noise.

As discussed above, the proposed KLD-EWMA methodology is effective in detecting traffic congestions, but its detec- 

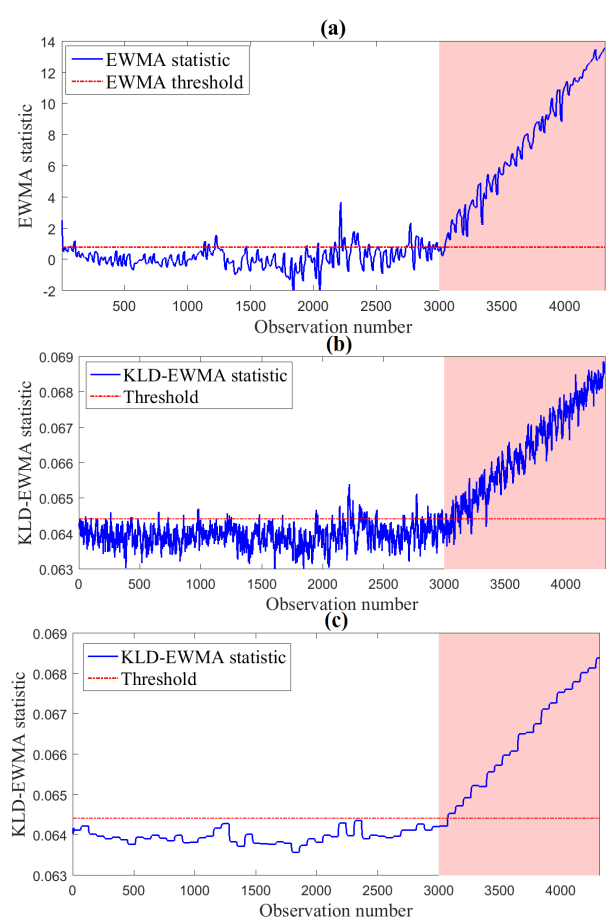

Fig. 14: Monitoring results from EWMA scheme (a), KLDEWMA scheme using the raw residuals (b) and the denoised residuals (c) in the case of gradual traffic congestion, noisy data example.

tion performance degrades under the noisy environment (increase in term of FAR and MDR). To overcome this limitation, we applied the KLD-EWMA approach to denoised residuals by wavelet-based multiscale filtering. Results show an important improvement when using filtered residuals than unfiltered residuals. We compared the efficiency of the proposed method with the conventional EWMA chart. This proposed approach scheme enjoys certain robustness properties when detecting traffic congestion in noisy data compared to the conventional EWMA scheme.

\section{CONCLUSION}

Nowadays, real-time monitoring of traffic congestion is desirable for a safe and reliable management of road traffic. A framework merging PWSL model and Luenberger observer, PWSL-LO, is proposed and used in the context of traffic congestion estimation and detection. Furthermore, a statistical approach for detecting traffic congestion based on KullbackLeibler distance (KLD) and EWMA monitoring scheme is proposed. The mixed KLD-EWMA approach uses the residuals for PWSL-LO to detect the presence of traffic congestions. To further enhance the sensitivity and the robustness to noises of the proposed approach, a multiscale filtering using wavelet has been applied to the residuals form PWSL-LO. Performance of the algorithm is evaluated using real traffic data from the fourlane SR-60 freeway in southern California. Results show the state estimation capabilities of the proposed observer and the improved sensitivity to faults and the robustness to noises of the proposed monitoring approach.
As future work, it would be interesting to merge benefit of Kalman filters, which are appropriate tools to deal with noisy environments, with the PWSL model to construct a robust approach for traffic density estimation. We plan also to work on a comparative study between hybrid approaches based on Kalman filters and observers.

\section{ACKNOWLEDGEMENT}

This publication is based upon work supported by King Abdullah University of Science and Technology (KAUST), Office of Sponsored Research (OSR) under Award No: OSR2015-CRG4-2582.

\section{REFERENCES}

[1] S. M. Khan, K. C. Dey, and M. Chowdhury, "Real-time traffic state estimation with connected vehicles," IEEE Transactions on Intelligent Transportation Systems, vol. 18, no. 7, pp. 1687-1699, July 2017.

[2] E. Thonhofer, E. Luchini, and S. Jakubek, "A flexible, adaptive traffic network simulation with parameter estimation," Journal of Intelligent Transportation Systems, vol. 21, no. 1, pp. 63-77, 2017.

[3] S. Saponara and B. Neri, "Radar sensor signal acquisition and multidimensional $\mathrm{fft}$ processing for surveillance applications in transport systems," IEEE Transactions on Instrumentation and Measurement, vol. 66, no. 4, pp. 604-615, April 2017.

[4] T. Seo, A. M. Bayen, T. Kusakabe, and Y. Asakura, "Traffic state estimation on highway: A comprehensive survey," Annual Reviews in Control, vol. 43, pp. 128 - 151, 2017.

[5] B. Smith, W. Scherer, and J. Conklin, "Exploring imputation techniques for missing data in transportation management systems," Transportation Research Record: Journal of the Transportation Research Board, vol. 1836, pp. 132-142, 2003.

[6] M. Zhong, P. Lingras, and S. Sharma, "Estimation of missing traffic counts using factor, genetic, neural, and regression techniques," Transportation Research Part C: Emerging Technologies, vol. 12, no. 2, pp. 139 - 166, 2004.

[7] N. Messai, P. Thomas, D. Lefebvre, and A. El Moudni, "A neural network approach for freeway traffic flow prediction," in Control Applications, 2002. Proceedings of the 2002 International Conference on, vol. 2. IEEE, 2002, pp. 984-989.

[8] D. Ni and J. L. II, "Markov chain monte carlo multiple imputation using bayesian networks for incomplete intelligent transportation systems data," Transportation Research Record: Journal of the Transportation Research Board, vol. 1935, pp. 57-67, 2005.

[9] D. W. Xu, H. H. Dong, H. J. Li, L. M. Jia, and Y. J. Feng, "The estimation of road traffic states based on compressive sensing," Transportmetrica B: Transport Dynamics, vol. 3, no. 2, pp. 131-152, 2015.

[10] N. G. Polson and V. O. Sokolov, "Deep learning for short-term traffic flow prediction," Transportation Research Part C: Emerging Technologies, vol. 79, pp. 1 - 17, 2017.

[11] H. Tan, G. Feng, J. Feng, W. Wang, Y.-J. Zhang, and F. Li, "A tensor-based method for missing traffic data completion," Transportation Research Part C: Emerging Technologies, vol. 28, pp. 15 - 27, 2013, euro Transportation: selected paper from the EWGT Meeting, Padova, September 2009.

[12] B. Ran, H. Tan, Y. Wu, and P. J. Jin, "Tensor based missing traffic data completion with spatialtemporal correlation," Physica A: Statistical Mechanics and its Applications, vol. 446, pp. 54 - 63, 2016.

[13] A. Nantes, D. Ngoduy, A. Bhaskar, M. Miska, and E. Chung, "Realtime traffic state estimation in urban corridors from heterogeneous data," Transportation Research Part C: Emerging Technologies, vol. 66, pp. 99-118, 2016.

[14] H. Sutarto, R. K. Boel, and E. Joelianto, "Parameter estimation for stochastic hybrid model applied to urban traffic flow estimation," IET Control Theory \& Applications, vol. 9, no. 11, pp. 1683-1691, 2015.

[15] Y. Wang and M. Papageorgiou, "Real-time freeway traffic state estimation based on extended kalman filter: A general approach," Transportation Research Part B: Methodological, vol. 39, no. 2, pp. 141-167, 2005.

[16] J. Thai and A. M. Bayen, "State estimation for polyhedral hybrid systems and applications to the godunov scheme for highway traffic estimation," IEEE Transactions on Automatic Control, vol. 60, no. 2, pp. 311-326, Feb 2015 . 
[17] L. Mihaylova and R. Boel, in 2004 43rd IEEE Conference on Decision and Control (CDC) (IEEE Cat. No.04CH37601).

[18] H. Martin, Treibera; Dirk, "Reconstructing the spatio-temporal traffic dynamics from stationary detector data," Cooperative Transportation Dynamics, 052002.

[19] B. Piccoli, K. Han, T. L. Friesz, T. Yao, and J. Tang, "Second-order models and traffic data from mobile sensors," Transportation Research Part C: Emerging Technologies, vol. 52, pp. 32 - 56, 2015.

[20] C. Vivas, S. Siri, A. Ferrara, S. Sacone, G. Cavanna, and F. R. Rubio, "Distributed consensus-based switched observers for freeway traffic density estimation," in 2015 54th IEEE Conference on Decision and Control (CDC), Dec 2015, pp. 3445-3450.

[21] E. Thonhofer, T. Palau, A. Kuhn, S. Jakubek, and M. Kozek, "Macroscopic traffic model for large scale urban traffic network design," Simulation Modelling Practice and Theory, vol. 80, pp. 32 - 49, 2018.

[22] L. Muñoz, X. Sun, R. Horowitz, and L. Alvarez, "Piecewise-linearized cell transmission model and parameter calibration methodology," Transportation Research Record: Journal of the Transportation Research Board, no. 1965, pp. 183-191, 2006.

[23] A. Zeroual, N. Messai, S. Kechida, and F. Hamdi, "A piecewise switched linear approach for traffic flow modeling," International Journal of Automation and Computing, 2017.

[24] D. Luenberger, "An introduction to observers," IEEE Transactions on Automatic Control, vol. 16, no. 6, pp. 596-602, Dec 1971.

[25] J. J. . A. S. Vinodh, K., "Archives of control sciences," Archives of Control Sciences, vol. 23, no. 2, pp. 243-256, 2013.

[26] S. R. E. Datondji, Y. Dupuis, P. Subirats, and P. Vasseur, "A survey of vision-based traffic monitoring of road intersections," IEEE transactions on intelligent transportation systems, vol. 17, no. 10, pp. 2681-2698, 2016.

[27] M. Madakyaru, F. Harrou, and Y. Sun, "Improved data-based fault detection strategy and application to distillation columns," Process Safety and Environmental Protection, vol. 107, pp. 22-34, 2017.

[28] M. Z. Sheriff, F. Harrou, and M. Nounou, "Univariate process monitoring using multiscale shewhart charts," in Control, Decision and Information Technologies (CoDIT), 2014 International Conference on. IEEE, 2014, pp. 435-440.

[29] Y. Wang and M. Papageorgiou, "Real-time freeway traffic state estimation based on extended Kalman filter: a general approach," Transportation Research Part B: Methodological, vol. 39, no. 2, pp. 141-167, 2005.

[30] L. Mihaylova, R. Boel, and A. Hegyi, "Freeway traffic estimation within particle filtering framework," Automatica, vol. 43, no. 2, pp. 290-300, 2007.

[31] X. Sun, L. Muñoz, and R. Horowitz, "Highway traffic state estimation using improved mixture kalman filters for effective ramp metering control," in 42nd IEEE Conference on Decision and Control, vol. 6. IEEE, 2003, pp. 6333-6338.

[32] L. Zhang and X. Mao, "Vehicle density estimation of freeway traffic with unknown boundary demandsupply: an interacting multiple model approach," IET Control Theory and Applications, vol. 9, no. 13, p. 19891995, 2015.

[33] Z. Hidayat, Z. Lendek, R. Babuška, and B. DeSchutter, "Fuzzy observer for state estimation of the METANET traffic model," in 13th International IEEE Conference on Intelligent Transportation Systems (ITSC). IEEE, 2010, pp. 19-24.

[34] R. Jithin, B. Hareesh, and D. Lelitha, "Application of data mining techniques for traffic density estimation and prediction," Transportation Research Procedia, vol. 17, pp. 321 - 330, 2016.

[35] A. Constantinos and K. Haris, "Estimation of traffic dynamics models with machine-learning methods," Transportation Research Record: Journal of the Transportation Research Board, vol. 1965, pp. 103-111, 2006.

[36] A. Mammeri, T. Zuo, and A. Boukerche, "Extending the detection range of vision-based vehicular instrumentation," IEEE Transactions on Instrumentation and Measurement, vol. 65, no. 4, pp. 856-873, April 2016.

[37] S. S. Beauchemin, M. A. Bauer, T. Kowsari, and J. Cho, "Portable and scalable vision-based vehicular instrumentation for the analysis of driver intentionality," IEEE Transactions on Instrumentation and Measurement, vol. 61, no. 2, pp. 391-401, Feb 2012.

[38] F. Mehboob, M. Abbas, and R. Jiang, "Traffic event detection from road surveillance vide os based on fuzzy logic," in SAI Computing Conference (SAI), 2016. IEEE, 2016, pp. 188-194.

[39] G. D. Leo, A. Pietrosanto, and P. Sommella, "Metrological performance of traffic detection systems," IEEE Transactions on Instrumentation and Measurement, vol. 58, no. 9, pp. 3199-3206, Sept 2009.
[40] W. Wang, S. Chen, and G. Qu, "Incident detection algorithm based on partial least squares regression," Transportation Research Part C: Emerging Technologies, vol. 16, no. 1, pp. 54-70, 2008.

[41] A. Troiano, E. Pasero, and L. Mesin, "New system for detecting road ice formation," IEEE Transactions on Instrumentation and Measurement, vol. 60, no. 3, pp. 1091-1101, March 2011.

[42] K. Y. Chan and T. S. Dillon, "On-road sensor configuration design for traffic flow prediction using fuzzy neural networks and taguchi method," IEEE Transactions on Instrumentation and Measurement, vol. 62, no. 1, pp. 50-59, Jan 2013.

[43] S. S. M. Ali, B. George, L. Vanajakshi, and J. Venkatraman, "A multiple inductive loop vehicle detection system for heterogeneous and laneless traffic," IEEE Transactions on Instrumentation and Measurement, vol. 61, no. 5, pp. 1353-1360, May 2012.

[44] C. F. Daganzo, "The cell transmission model, part II: Network traffic," Transportation Research Part B: Methodological, vol. 29, no. 2, pp. 79-93, 1995.

[45] F. Harrou, Y. Sun, and M. Madakyaru, "Kullback-leibler distance-based enhanced detection of incipient anomalies," Journal of Loss Prevention in the Process Industries, vol. 44, pp. 73-87, 2016.

[46] L. Pardo, Statistical inference based on divergence measures. CRC press, 2005.

[47] F. Harrou and M. N. Nounou, "Monitoring linear antenna arrays using an exponentially weighted moving average-based fault detection scheme," Systems Science \& Control Engineering: An Open Access Journal, vol. 2, no. 1, pp. 433-443, 2014.

[48] D. Montgomery, Introduction to statistical quality control. John Wiley \& Sons, 2007.

[49] G. Dervisoglu, G. Gomes, J. Kwon, R. Horowitz, and P. Varaiya, "Automatic calibration of the fundamental diagram and empirical observations on capacity," in Transportation Research Board 88th Annual Meeting, vol. 15,2009

[50] A. Zeroual, N. Messai, S. Kechida, and F. Hamdi, "Calibration and Validation of a Switched Linear Macroscopic Traffic Model," in 3rd International Conference on Control, Engineering \& Information Technology CEIT2015, no. 1. IEEE, 2015, pp. 25-27.

[51] F. Harrou, M. Madakyaru, Y. Sun, and S. Khadraoui, "Improved detection of incipient anomalies via multivariate memory monitoring charts: application to an air flow heating system," Applied Thermal Engineering, vol. 109, pp. 65-74, 2016. 